\title{
Competitive hydrogen bonds associated with the effect of primycin antibiotic on oleic acid as a building block of plasma membranes
}

\begin{abstract}
Eszter Virág ${ }^{1,3}$, Miklós Pesti ${ }^{1}$ and Sándor Kunsági-Máté ${ }^{2}$
Interaction of primycin antibiotic with oleic acid was investigated to understand the effect of primycin on lipid membranes at a molecular level. The thermodynamic parameters of the complex formation were determined by photoluminescence studies. Results highlight the presence of two interactions between the interacted species according to the cases in which one or two hydrogen bonds stabilize the molecular complexes. Although both interactions result in similar Gibbs-free enthalpy change at room temperature, the enthalpy and entropy changes associated with the formation of single or double hydrogen bonds are quite different. Quantum chemical and anisotropy decay studies validated that the formation of a single or double hydrogen bond between these species is driven by entropy in the former or enthalpy in the latter case. Owing to the resultant quite different temperature dependence of these two interactions, after detailed examinations in a cellular environment, this property could have importance in application of primycin on differently fevered bodies.
\end{abstract}

The Journal of Antibiotics (2010) 63, 113-117; doi:10.1038/ja.2009.130; published online 15 January 2010

Keywords: antimycotic; interactions; membrane; oleic acid; primycin

\section{INTRODUCTION}

There has been a significant increase in the number of opportunistic fungal and bacterial infections in recent decades. ${ }^{1}$ Primycin is one among the few antibiotics that exhibits a broad antimicrobial spectrum and it proved to be effective against both the known Gram-positive bacteria and pathogenic yeasts and filamentous fungi, such as Staphylococcus, Streptococcus, Mycobacterium, Candida, Aspergillus, etc. At elevated concentrations, it also inhibits Gramnegative bacteria, including antibiotic-resistant and polyresistant strains. ${ }^{2}$ In medical practice, primycin containing Ebrimycin gel has been successfully applied in the prevention of burn infections, in the treatment of cacogenic processes, bacterially infected trophic ulcers, postoperative suppurations, inflammation of anorectum, as well as in preoperative and postoperative prevention..$^{2-6}$ It is a nonpolyene macrolide lactone antibiotic complex, ${ }^{7}$ which is strongly surface active, thermostable and absorptive ${ }^{8}$ and exerts a specific effect on plasma membrane permeability by inducing the leakage of $\mathrm{K}^{+}$ions from red blood cells. In addition, no lysis of cell wall-free protoplast of Candida albicans was observed. ${ }^{9}$ In spite of intensive research, the mechanism of the effect of primycin is not completely revealed yet. On the basis of these preliminary results, it was assumed that primycin interacts with plasma membrane of different types of cells and this interaction is specific to fatty acids that are one of the main components of biological membranes.
The aim of this study was to investigate the temperature dependence of primycin-oleic acid interactions with possible applications of the results in pharmaceutical research. Owing to the importance of temperature, the entropy change further to the enthalpy change of the interaction is studied in detail. The highly sensitive photoluminescence (PL) method and quantum-chemical studies are applied for these investigations.

\section{RESULTS AND DISCUSSION}

Oleic acid was selected for these studies because it is the main component (35\%) of fatty acids of the most important human pathogen C. albicans. ${ }^{10}$ Figure 1 shows the PL spectra of oleic acid in the presence of various primycin concentrations at $295.16 \mathrm{~K}$. The weak fluorescence of oleic acid, probably because of the $\mathrm{C}=\mathrm{C}$ double bond, exists in its skeleton. It can be clearly seen in Figure 1 that the presence of primycin reduces the PL signal significantly. This property is well known to be associated with the increased nonradiative energy loss of a fluorescent molecule by assisting coordinated nonfluorescent primycin molecules. ${ }^{11}$ In accordance with our previous observations on weak host-guest interactions, ${ }^{12,13}$ the decrease observed in the PL signal reflects the concentration of the oleic acid-primycin complexes.

The fluorescence spectra show a complex structure and suggest the presence of different fluorescent species in the solutions. To obtain

${ }^{1}$ Faculty of Sciences, Department of General and Environmental Microbiology, University of Pécs, Pécs, Hungary; ${ }^{2}$ Faculty of Sciences, Department of General and Physical Chemistry, University of Pécs, Pécs, Hungary and 3PannonPharma Ltd, Pécsvárad, Hungary

Correspondence: Dr S Kunsági-Máté, Department of General and Physical Chemistry, University of Pécs, Ifjúság 6., Pécs, H-7624, Hungary.

E-mail: kunsagi@gamma.ttk.pte.hu

Received 6 October 2009; revised 6 December 2009; accepted 10 December 2009; published online 15 January 2010 


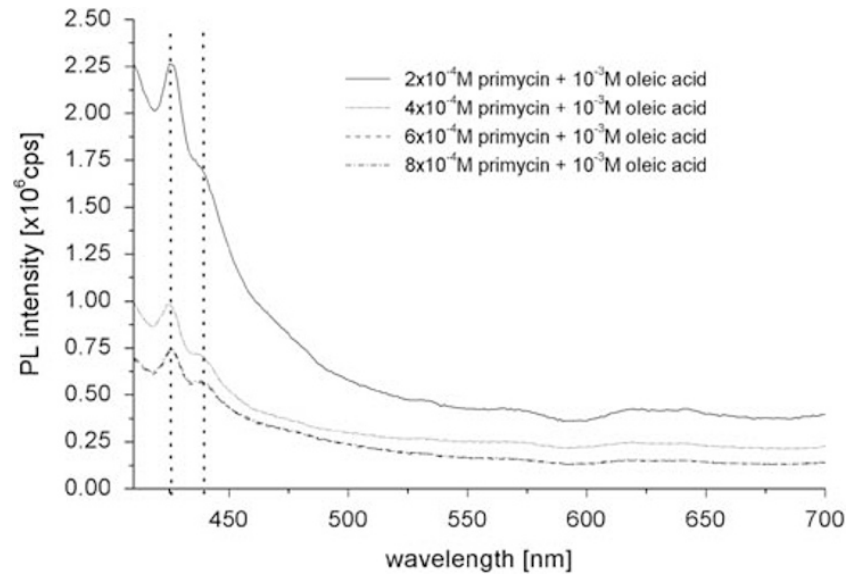

Figure 1 Representative photoluminescence $(\mathrm{PL})$ spectra of oleic acid in the presence of different concentrations of primycin. Spectra recorded at 295.16 K. Dotted lines highlight the peaks used for determination of equilibrium constants.

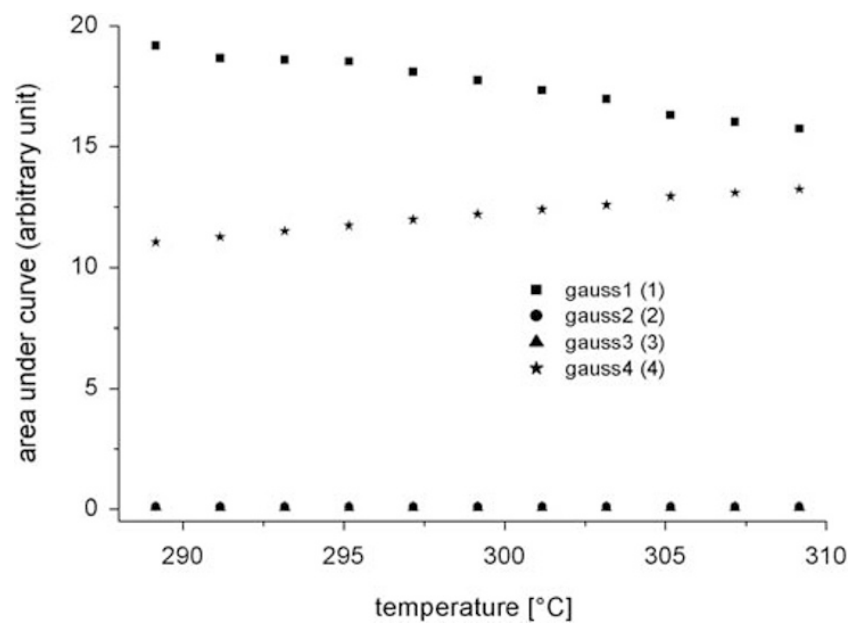

Figure 2 Temperature dependence of the different species present in the solution of oleic acid $\left(10^{-3} \mathrm{M}\right)$ and primycin $\left(4 \times 10^{-4} \mathrm{M}\right)$.

quantitative results on the possible forms of oleic acid-primycin complexes, emission spectra were decomposed to Gauss functions. To do so, PL intensities were plotted first against energy, that is, PL intensity was plotted as a function of wave numbers $\left(\mathrm{cm}^{-1}\right)$. Thereafter, the area associated with individual Gauss functions was plotted against temperature. Figure 2 shows the temperature dependence of the Gauss functions used for the deconvolution of the PL spectra. Results suggest the presence of two species in the solutions: according to the change in their equilibrium with temperature, the concentration of a species increases, whereas the concentration of another species decreases at elevated temperatures. To obtain quantitative data on the thermodynamics of the possible chemical equilibriums present in this system, the equilibrium constant was determined first by the Benesi-Hildebrand method using the two series of data associated with the two different temperature dependences as mentioned above.

According to the Benesi-Hildebrand equation, if the complex formed possesses 1:1 stoichiometry, the plot of the reciprocal PL change against the reciprocal concentration of one of the reactants should show a

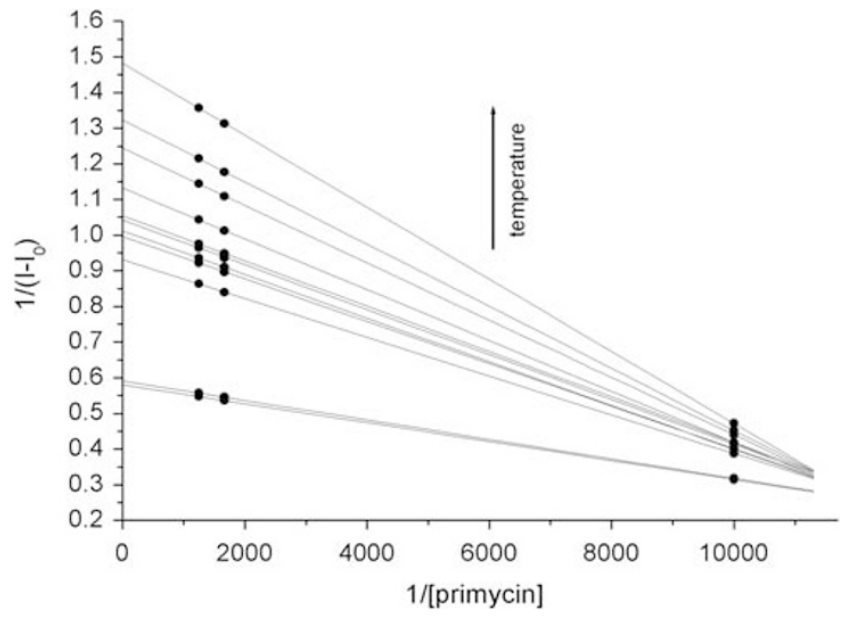

Figure 3 Benesi-Hildebrand plot of the photoluminescence $(P L)$ signal observed for oleic acid-primycin complexes.

straight line. Therefore, by linearly fitting these data points, the equilibrium constant can be determined using the following equation:

$$
\frac{1}{I-I_{0}}=\frac{1}{a} \cdot \frac{1}{[\mathrm{p}]} \cdot \frac{1}{K}+\frac{1}{a}
$$

In this equation, [p] and [a] represent the actual and analytical concentration of primycin and oleic acid, respectively. $I$ is the intensity of the samples containing both the reactants, and $I_{0}$ is the PL intensity of the oleic acid solution in the absence of primycin. Figure 3 shows the Benesi-Hildebrand plot of the PL signal at different temperatures. The increased slope at an elevated temperature shows decreased stability of the complexes.

The temperature dependence of the equilibrium constants was applied to determine the enthalpy and entropy changes of the association reaction using the van't Hoff theory. According to the van't Hoff equation, the logarithm of the equilibrium constants was plotted against the reciprocal temperature (Figure 4):

$$
\ln K=-\frac{\Delta H}{R} \cdot \frac{1}{T}+\frac{\Delta S}{R}
$$

Enthalpy change can be obtained from the slope, whereas entropy change can be determined from the intercept of the line fitted to the experimental data. Results are summarized in Table 1.

Results highlight the presence of two chemical equilibriums between oleic acid and primycin. These two processes show significantly different thermodynamics; therefore, they cannot be considered as opposite reactions of each other. Both reactions finish with similar Gibbs-free enthalpy change, resulting in complexes with similar stability. The strength of these interactions suggests the participation of hydrogen bonds in complex formation. However, whereas reaction 1 is forced preferably by the enthalpy change, reaction 2 is driven by the increased entropy during complex formation. The significant difference obtained in the entropy term is probably due to the following known molecular processes: The two reactant molecules have to at least lose their solvation shell before association. The movement of small solvent molecules increases after leaving the solvation shell. ${ }^{14}$ This process increases the entropy. However, association of the reactants decreases entropy because of the reduced freedom 


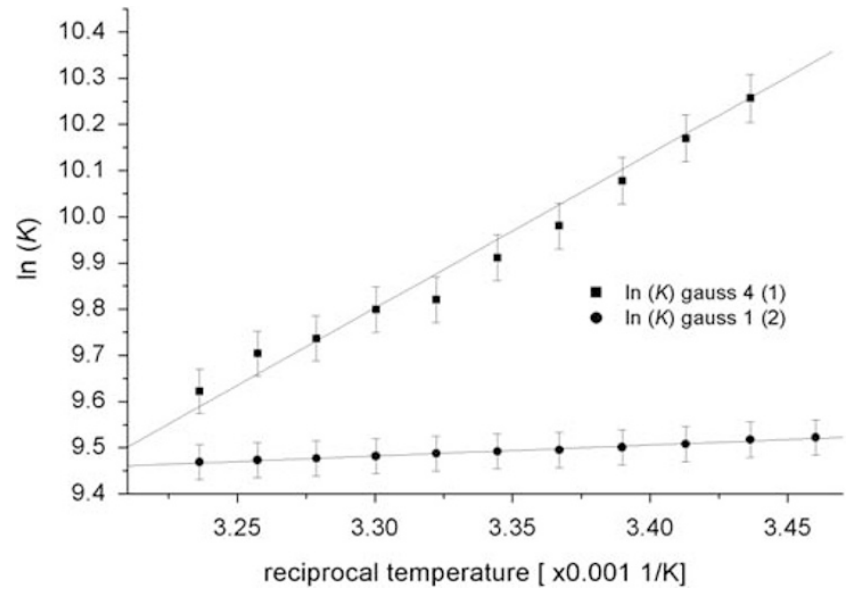

Figure 4 van't Hoff plot of the equilibrium constants $K$ determined by the Benesi-Hildebrand method. Thermodynamic parameters were determined from the slope and the intercept of the fitted line according to Equation (2).

Table 1 Thermodynamic parameters calculated by applying the van't Hoff theory for the two reactions identified according to the oleic acid-primycin association

\begin{tabular}{lrr}
\hline & Reaction 1 & Reaction 2 \\
\hline$\Delta \mathrm{H}\left(\mathrm{kJ} \mathrm{mol}^{-1}\right)$ & -27.77 & -8.08 \\
$\Delta \mathrm{S}\left(\mathrm{J} \mathrm{mol}^{-1} \mathrm{~K}^{-1}\right)$ & -10.14 & 53.94 \\
$\Delta \mathrm{G}\left(20^{\circ} \mathrm{C}\right)\left(\mathrm{kJ} \mathrm{mol}^{-1}\right)$ & -25.80 & -23.88 \\
\hline
\end{tabular}

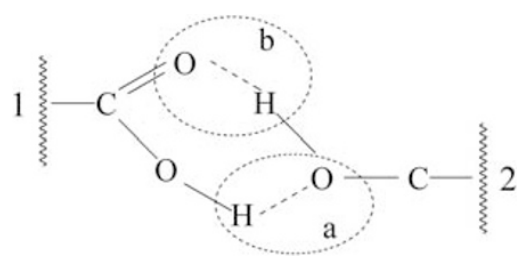

Figure $5 \mathrm{~A}$ schematic view of the possible formation of the $\mathrm{H}$-bonds between oleic acid (1) and primycin (2). The presence of two interactions between the interacted species: single (a) or double $(\mathbf{a}, \mathbf{b})$ hydrogen bonds can stabilize the molecular complexes.

of individual reactant molecules compared with their freedom before association. This process decreases the entropy. The competition of these two opposite processes determines the sum of change of the entropy term. In addition to the general situations described above, in our particular case, we describe this observation by assuming the following processes at a molecular level. Oleic acid reaches primycin molecules by its carboxyl group and this carboxyl group interacts with the $-\mathrm{OH}$ groups of primycin molecules. As a result, two situations can be formed (Figure 5): (1) a single hydrogen bond formed between the $-\mathrm{OH}$ groups of the carboxyl group of the oleic acid and one from those of primycin (Figure 5, case a); or (2) a hydrogen bond formed between the $\mathrm{OH}$ bond of primycin and the $\mathrm{O}$ atom of the carboxyl group of oleic acid (Figure 5, case b), in addition to the bond formed already in the former (1) case. Both situations assume a partial loss of the solvation shell, a process that gains entropy. This increased entropy decreases during the association of the two reactants. However, the freedom of the system reduces much more when more flexible double hydrogen bonds are formed during reaction 1 between the reactants, compared with the situation in which a single hydrogen bond forms according to reaction 2 . As a result, the enthalpy change is lower in reaction 2 (single hydrogen bond) than in reaction 1 (double hydrogen bond). Furthermore, entropy increases or decreases as single or double hydrogen bonds stabilize the complexes, respectively.

Figure 4 clearly shows that owing to the different temperature dependences of the free enthalpy change, the lines fitted to the experimental data are crossed at a significant temperature of $\sim 314 \mathrm{~K}$. This means that above and below this temperature, the driving force of the molecular association is quite different. Whereas above this temperature, enthalpy changes, below this temperature, entropy changes, which has a determinant role in the formation of oleic acid-primycin complexes.

To support the ideas described above, further theoretical and experimental investigations were carried out. As the primycin molecule possesses several $-\mathrm{OH}$ groups, quantum-chemical analysis was performed at the density functional theory (DFT) level to observe the possible target sites at which the carboxyl group of oleic acid can form hydrogen bonds with primycin. This calculation validates the fact that interactions between the associated species occur at the two secondary hydroxylic groups of primycin, that is, at the 2-hydroxy substituent of the side chain bearing guanidinium moiety and at the $15-\mathrm{OH}$ group in the allylic position (Figures 5 and 6). The interaction energy associated with those sites is the same, exactly -12.46 and $-33.16 \mathrm{~kJ} \mathrm{~mol}^{-1}$ for the single and double hydrogen bonds, respectively. These values are close to the enthalpy change observed experimentally and deviances can be originated from the theoretical approximation of the dielectric background: theoretical calculations are performed by the polarizable continuum method, whereas experiments are conducted in DMSO (dimethyl sulfoxide, $\varepsilon_{r}=48$ ). However, it is to be mentioned that, because of the pronounced importance of hydrogen bonds in the interaction, and considering the participation of the carboxyl group of oleic acid in the interaction, the local $\mathrm{pH}$ in a physiological environment could affect the stability of primycin-oleic acid complexes. Unfortunately, this characteristic cannot be discussed in this context because of the insolubility of oleic acid in water. Anyway, the very weak acidity of oleic acid ${ }^{15}$ suggests that no considerable $\mathrm{pH}$ dependence of protonation of the carboxyl moiety can be estimated in the physiological environment.

To support our description of the quite different entropy change observed for the two association reactions, an anisotropy decay study was conducted. Anisotropy decay was measured at $298.16 \mathrm{~K}$ and excitation and emission wavelengths were chosen according to the deconvolution of the spectra by Gauss functions: excitation was performed at $390 \mathrm{~nm}$, whereas emission wavelength was set to $426 \mathrm{~nm}$ (reaction 1) and $440 \mathrm{~nm}$ (reaction 2). Anisotropy shows $890 \pm 30$ ps decay in the former case, whereas this value reduces significantly in the latter case to $673 \pm 30$ ps. These data clearly show the much faster rotational diffusion of oleic acid when it bound to primycin by assistance of a single hydrogen bond compared with the double-bonded situation. These results are in agreement with our observation above that the entropy change highlights a higher reduction in freedom during association reaction 1 in comparison with reaction 2 .

\section{EXPERIMENTAL SECTION}

\section{Chemicals}

Primycin (molecular weight $=1127.25 \mathrm{~g} \mathrm{~mol}^{-1}$, average mass) was provided by the manufacturer (Pannonpharma, Pécsvárad, Hungary) as standard powder. 
a<smiles>CCCCCCCC/C=C\CCCCCCCCCC(=O)O</smiles>

b

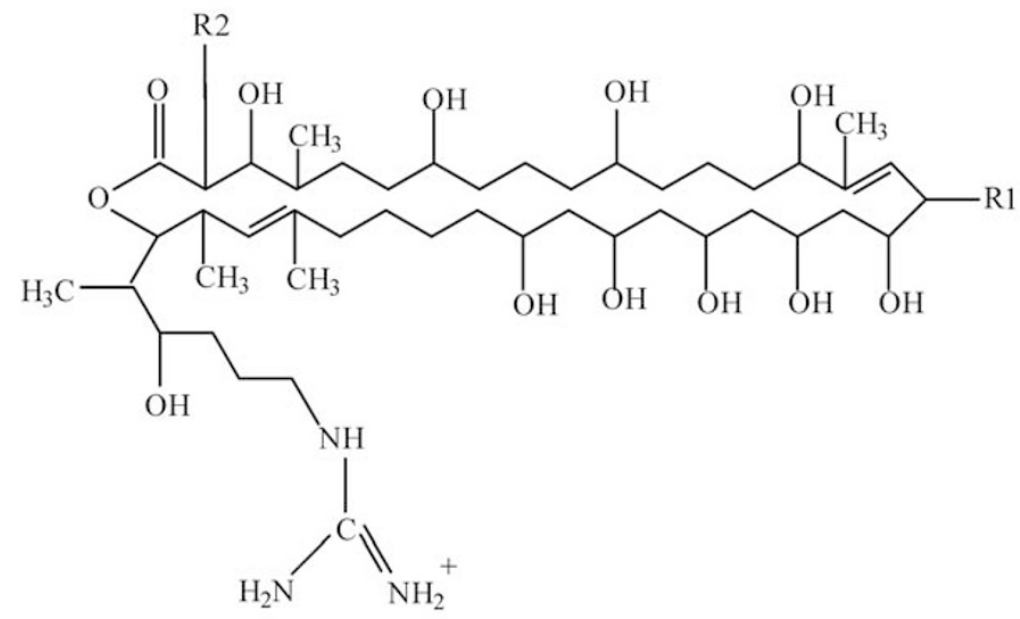

c

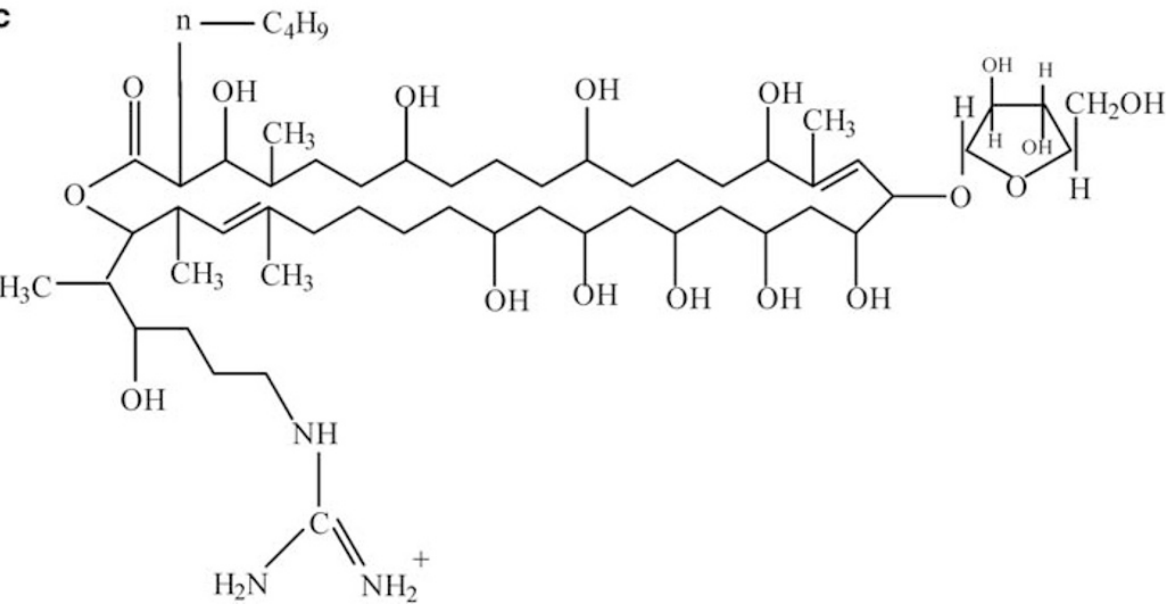

Figure 6 Structures of species the interaction of which was investigated in this work: oleic acid (a), general structure of primycin (b) and main component of primycin, A1 (c).

On the basis of information obtained from documents, the content of active agent in the sample of primycin was $86503 \mathrm{U} \mathrm{mg}^{-1}$. It was dissolved in DMSO (HPLC grade, Sigma-Aldrich, St Louis, MO, USA) at a concentration of $6.4 \mathrm{mg} \mathrm{ml}^{-1}$. The stock solution was stored at $4{ }^{\circ} \mathrm{C}$. Oleic acid (molecular weight $=282.46 \mathrm{~g} \mathrm{~mol}^{-1}$, Sigma-Aldrich) was dissolved in DMSO and stored at $-20^{\circ} \mathrm{C}$ (Figure 6) The purity of both specimens was better than $95 \%$.

The composition of primycin: On the basis of layer chromatography, primycin was identified as a group of materials consisting of more then 20 derivatives. Analyses performed by column chromatography showed that primycin molecules possess functional groups at their terminal positions as listed below. Although the mixture of primycin has nine components, they can be classified into three subgroups (A, B and C) according to the R1 substituent connected to the lactone ring. Accordingly, in group A, arabinose; in group B, hydrogen; and in group $\mathrm{C}$, hydroxil are coordinated to the lactone ring. Each subgroup is a mixture of homologs (A1, A2, A3, B1, B2, B3, C1, C2, C3) depending on the R2 substituent. They are 1-butyl, 2-pentyl or 3-hexyl groups (Table 2) (Figure 6b). The major constituent is Al (molecular weight $=1078$, see Figure $6 \mathrm{c}$ ). This derivative is presented in the mixture with $75 \mathrm{wt} \%$. The average molecular weight of primycin was applied during sample preparation and in data evaluation. This approximation results in $<0.2 \%$ s.d. in thermodynamic
Table 2 The functional groups at the terminal positions of primycin

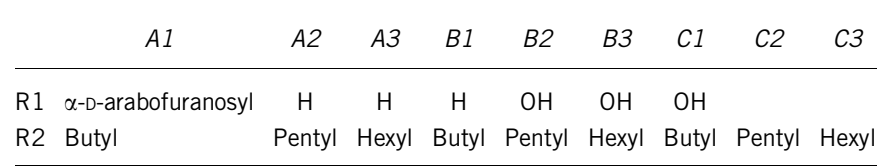

parameters. In addition, we have to highlight here that all derivatives of primycine that existed in the mixture possess those structural segments (2-hydroxy substituent of the side chain bearing guanidinium moiety and the 15-OH group in allylic position) that were found to be determinant in the interaction of primycine with oleic acid.

\section{Sample preparation}

Experiments were carried out by PL studies and data evaluation was performed by the Benesi-Hildebrand method. ${ }^{16}$ For measurements, stock solutions of $0.002 \mathrm{M}$ of oleic acid and $0.0016 \mathrm{M}$ of primycin were prepared in DMSO. Stock 
solutions of oleic acid and of primycin were diluted at four different molar ratios. Whereas the concentration of oleic acid was kept constant $(0.001 \mathrm{M})$ in the samples, the concentration of primycin was varied from $2 \times 10^{-4}$ to $8 \times 10^{-4} \mathrm{M}$. To avoid discrepancies in data evaluation from the possible formation of oleic acid-primycin complexes with higher stoichiometry, measurements were repeated with doubled primycin concentrations.

\section{Instruments}

Steady-state PL spectra were recorded on a Fluorolog $\tau 3$ spectrofluorometer (Jobin-Yvon/SPEX, Longjumeau, France). For data collection, the photoncounting method with a $0.2 \mathrm{~s}$ integration time was used. Excitation and emission bandwidths were set to $5 \mathrm{~nm}$. Front-face detection was used to eliminate the inner-filter effect. The excitation wavelength was $390 \mathrm{~nm}$, and emission spectra were recorded in the $410-700 \mathrm{~nm}$ range. Measurements were taken at 11 different temperatures within the $289.16-309.16 \mathrm{~K}$ range by 2-K step. PL spectra of the samples described above were recorded, and the average of 10 scans was used for data evaluation by DataMax 2.20 software (Jobin-Yvon).

\section{Calculation procedure}

Anisotropy decay studies were measured by the above system using a phasefluorometry setup. The modulation of the excitation beam was varied within $50-200 \mathrm{MHz}$, and Glann-Thompson prisms were used as polarizers. Work function of the fluorometer was recorded using $\mathrm{MgO}$ as reference sample.

Equilibrium conformations of the individual oleic acid and primycin (derivative A1) molecules were determined as follows: After determining equilibrium conformations at the semi-empirical AM1 level, geometries were refined by B3LYP/DFT/6-31++G calculations. The Berny geometry optimization method was used for the investigation of conformers. To select possible sites of the interaction, reaction channels were analyzed by the HyperChem 7.1 (HyperCube, Gainesville, FL, USA) code ${ }^{17}$ at the semi-empirical AM1 level. Interaction energy was then calculated by the B3LYP/DFT/6-31++G method. During these calculations, only the moieties of molecules involved in the interactions were allowed to relax (primycin: 2-hydroxy substituent of the sidechain bearing guanidinium moiety and the $15-\mathrm{OH}$ group in allylic position; oleic acid: carboxyl moiety). Other parts of molecules were frozen during the calculation of interaction energy. Solvents were considered by the method. DFT calculations were performed using the Gaussian 03 program package (Gaussian, Wallingford, CT, USA). ${ }^{18}$

\section{CONCLUSION}

The interaction of oleic acid with primycin antibiotic shows a complex character that is preferably based on the formation of molecular complexes stabilized by single or double hydrogen bonds. Thermodynamic parameters derived by PL studies show a similar free enthalpy change for both cases at room temperature. However, quite different enthalpy and entropy changes associated with different interactions. This characteristic results in a different temperature dependence of the two processes that stabilize the oleic acid-primycin complexes. As an important consequence, the reaction mechanism of primycin applied on differently fevered bodies can differ significantly. However, to understand the exact mode of action of primycin, further investigations with better simulation of the living cell environment are planned with the main components of plasma membrane, such as phospholipids and sterols.

\section{ACKNOWLEDGEMENTS}

This research was supported in part by Grant no. RET-08/2005 and by PannonPharma (Pécsvárad, Hungary). SK-M expresses gratitude to the Hungarian Academy of Sciences for a Bolyai János Research Fellowship.

1 Singh, N. Trends in the epidemiology of opportunistic fungal infections: predisposing factors and the impact of antimicrobial use practices. Clin. Infect. Dis. 33, 1692-1696 (2001).

2 Nógrádi, M. Primycin (Ebrimycin)—a new topical antibiotic. Drugs Today 24, 563-566 (1988)

3 Papp, T., Menesi, L. \& Szalai, I. Experiences in the Ebrimycin gel treatment of burns. Ther. Hung. 38, 125-128 (1990).

4 Biro, J. \& Varkonyi, V. Ebrimycin gel in the treatment of pyodermas and bacterial secondary infections. Ther. Hung. 35, 136-139 (1987).

5 Meszaros, C. \& Vezekenyi, K Use of Ebrimycin gel in dermatology. Ther. Hung. 35, 77-79 (1987)

6 Balint, B. Favourable observations with Ebrimycin gel in the outpatient department of surgery. Ther. Hung. 35, 140-142 (1987).

7 Alberhart, J., Fehr, T., Jain, R. C., de Mayo, P. \& Motl, O. J. Primycin. Am. Chem. Soc. 92, 5816-5817 (1970).

8 Vályi-Nagy, T. \& Daróczy, A. Effects of primycin on the synthesis of tryptophan pyrrolase. Biochem. Pharmac. 16, 1051-1055 (1967).

9 Blaskó, K., Györgyi, S. \& Horváth, I. Effect of primycin on monovalent cation transport of erythrocyte membrane and lipid bilayer. J. Antibiot. 32, 408-413 (1979).

10 Pesti, M., Horváth, L., Vígh, L. \& Farkas, T. ESR determination of plasma membrane order parameter, lipid content and phase transition point in Candida albicans sterol mutants. Acta. Microbiol. Hung. 32, 305-313 (1984)

11 Kunsági-Máté, S. et al. Complexation of phenols by calix[4]arene diethers in a low-permittivity solvent self-switched complexation by 25,27-dibenzyloxycalix[4]arene. J. Phys. Chem. B 111, 7218-7223 (2007).

12 Kunsági-Máté, S., Kumar, A., Sharma, P., Kollár, L. \& Nikfardjam, M. P. Effect of molecular environment on the formation kinetics of complexes of malvidin-3-0-glucoside with caffeic acid and catechin. J. Phys. Chem. B 113, 7468-7473 (2009).

13 Kunsági-Máté, S., Csók, Z. S., Tuzi, A. \& Kollár, L. Permittivity-dependent entropy driven complexation ability of cone and paco tetranitro-calix[4]arene toward para-substituted phenols. J. Phys. Chem. B 112, 11743-11749 (2008).

14 Kunsági-Máté, S. \& Iwata, K. Effect of cluster formation of solvent molecules on the preferential solvatation of anthracene in binary alcoholic solutions. Chem. Phys. Lett. 473, 284-287 (2009)

15 Liu, X.- M. \& Wang, L.- S. A one-pot synthesis of oleic acid end-capped temperatureand $\mathrm{pH}$-sensitive amphiphilic polymers. Biomaterials 25, 1929-1936 (2004).

16 Benesi, H. \& Hildebrand, J. A spectrophotometric investigation of the interaction of iodine with aromatic hydrocarbons. J. Am. Chem. Soc. 71, 2703-2707 (1949).

17 HyperCube. HyperChem Professional 7 (HyperCube, Gainesville, FL, 2002).

18 Frisch, M. J. et al. Gaussian 03, Revision C.02 (Gaussian, Wallingford, CT, 2004). 